\title{
Exploring Neural Cell Dynamics with Digital Holographic Microscopy
}

\author{
Pierre Marquet ${ }^{1,3}$, Pascal Jourdain ${ }^{1,3}$, Daniel Boss ${ }^{1,3}$, Christian Depeursinge ${ }^{2}$, Pierre J. Magistretti ${ }^{1,3,4}$ \\ 1- Centre de Neurosciences Psychiatriques, Département de psychiatrie DP-CHUV, Site de Cery, 1008 Prilly-Lausanne, Switzerland \\ 2-Ecole Polytechnique Fédérale de Lausanne, Laboratoire d'Optique Appliquée, 1015 Lausanne, Switzerland \\ 1-Brain Mind Institute, École Polytechnique Fédérale de Lausanne, 1015 Lausanne, Switzerland \\ 4 Chemical Life Sciences and Engineering Division, King Abdullah University of Science \& Technology Thuwal 23955-6900, Saudi Arabia.
}

Authore-mail address: pierre.marquet@chuv.ch

Abstract: This paper describes how digital holographic microscopy, as a powerful quantitative phase technique, can non-invasively measure cell dynamics and especially resolve local neuronal network activity by performing simultaneous multiple site optical recording.

OCIS codes: $(090.0090) ;(180.0180)$

\section{Introduction to the style guide, formatting of main text, and page layout}

The quantitative phase microscopy technique we have developed, is an interferometric approach based on the holographic principle [1]. Briefly, from a single recorded hologram, quantitative phase images of transparent specimen can be fully reconstructed by a numerical process. This numerical processing of holograms presents the great advantage of offering not only the possibility to reconstruct quantitative phase image but also to achieve a numerical compensation for experimental noise (time drift, vibration, etc.) and a-priori any type of aberration including apparent spherical aberration resulting from the mismatch between the reference and object wave. It results a simplified and easy-to-operate interferometric technique, called digital holographic quantitative phase microscopy (DH-QPM) (Fig.1 ) having the phase sensitivity to observe cell dynamics processes with a nanometric axial accuracy [2].
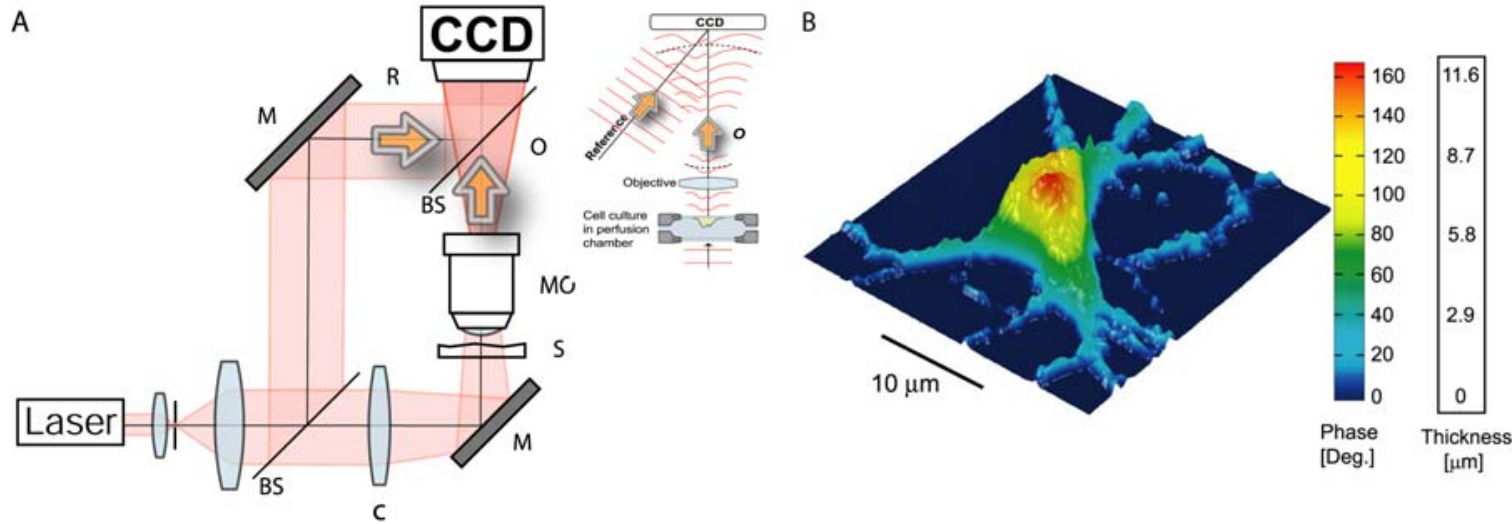

Fig. 1 DHM-QP of living mouse cortical neurons in culture. A) DH-QPM transmission set-up. Inset: Schematic representation of cultured cells mounted in a closed perfusion chamber and trans-illuminated B) 3D perspective quantitative phase image in false colors of a living neuron in culture. Each pixel represents OPD induces by the cell (with a sensitivity corresponding to a few tens of nanometers). By using the measured mean value of the neuronal cell body refractive index, resulting from a decoupling procedure, scales (at right), which relate OPL (Deg) to morphology in the $Z$-axis $(\mu \mathrm{m})$, can be constructed.

Practically, the reconstructed quantitative phase images provide accurate measurement of the phase retardation or optical path difference (OPD) induced by a given transparent specimen on the transmitted wave front. This phase retardation $\phi$, which arises from a mismatch $\Delta \mathrm{n}$ between the intracellular integral refractive index (RI) $n_{c}$, defined as the mean cellular RI along the optical axis, and the RI of the extracellular medium $n_{s}$ is given by equation 


$$
\phi=\frac{2 \pi}{\lambda} d\left(n_{c}-n_{s}\right) \propto d \Delta n=O P D
$$

Here $\lambda$, denotes the wavelength, $\mathrm{d}$ cellular thickness.

This optical phase retardation can be regarded as a powerful endogenous contrast agent, as it contains information about both the thickness and the RI of the transparent sample. As far as biological cells are concerned, the origin of the "phase signal" resides in the refractive index difference generated by the presence of organic molecules: proteins, DNA, organelles, nuclei present in cells. Practically, important biophysical cell parameters can be calculated from the quantitative phase signal, including cell shape and absolute volume [3, 4], dry mass concentration [5], membrane mechanical properties [6] and permeability [3], transmembrane ionic currents [7] etc.

\section{Resolving local neural network activity}

A distinct feature of the nervous tissue is the intricate network of synaptic connections among neurons of diverse phenotypes. Although initial connections are formed largely through molecular mechanisms, there is little doubt that electrical activities plays a pivotal role in the development and integrative functions of neural networks/circuits. However, our understanding of how electrical activity affects the structure and function of neuronal network is very limited. To be able to non-invasively resolve local neuronal network activity is thus a prerequisite for comprehensive studies of the relationships between spatiotemporal activity patterns and neuronal network development and information processing.

With respect to the study of neuronal activity, electrophysiological approaches, in particular voltage clamp and patch-clamp techniques have permitted a major breakthrough in the understanding of these relationships. However, patch clamp is still a highly invasive laborious process requiring precise micromanipulations and a high degree of operation skills, which generally impose the recording of voltages on a limited number of cells that form a neuronal network.

On the other hand, it is well known that neuronal activity induces modifications of its own intrinsic optical properties [8]. Practically, the study of local application of the excitatory neurotransmitter glutamate $(500 \mu \mathrm{M}, 200$ $\mathrm{ms}$ ) on primary cultures of mouse cortical neurons with a set-up combining electrophysiology and DH-QPM, has allowed to reveal that one of the mechanisms inducing the activity-related modifications of the neuronal intrinsic optical properties are transmembrane water movements, which drastically modifies the intracellular RI. Consequently, a mathematical relationship, involving some cell morphology parameters, as well as a parameter " $\varepsilon$ " $[\mathrm{ml} / \mathrm{C}]$ representing the volume of the water movement associated with the net charge transported through the cell membrane, has been established between net transmembrane currents and the phase signals [7]. This result, allowing to perform simultaneous multiple site optical recording of transmembrane currents, opens thus the possibility to noninvasively resolve local neuronal network activity with DH-QPM.

\section{Acknowledgements}

This work was funded by the Swiss National Science Foundation (SNSF, grant no. CR3213 132993)

\section{References}

1. D. Gabor, "A new microscopic principle," Nature 161, 777 (1948)

2. P. Marquet, B. Rappaz, P. J. Magistretti, E. Cuche, Y. Emery, T. Colomb, and C. Depeursinge, "Digital holographic microscopy: a noninvasive contrast imaging technique allowing quantitative visualization of living cells with subwavelength axial accuracy," Opt Lett 30, 468-470 (2005).

3. D. Boss, J. Kuehn, P. Jourdain, C. Depeursinge, P. J. Magistretti, and P. Marquet, "Measurement of absolute cell volume and osmotic water membrane permeability by real time dual wavelength holographic microscopy," J Biomed Opt in press(2013).

4. B. Rappaz, P. Marquet, E. Cuche, Y. Emery, C. Depeursinge, and P. J. Magistretti, "Measurement of the integral refractive index and dynamic cell morphometry of living cells with digital holographic microscopy," Opt Express 13, 9361 - 9373 (2005).

5. B. Rappaz, E. Cano, T. Colomb, J. Kuhn, C. Depeursinge, V. Simanis, P. J. Magistretti, and P. Marquet, "Noninvasive characterization of the fission yeast cell cycle by monitoring dry mass with digital holographic microscopy," J Biomed Opt 14(2009).

6. D. Boss, A. Hoffmann, B. Rappaz, C. Depeursinge, P. J. Magistretti, D. Van de Ville, and P. Marquet, "Spatially-Resolved Eigenmode Decomposition of Red Blood Cells Membrane Fluctuations Questions the Role of ATP in Flickering," Plos One 7(2012).

7. P. Jourdain, D. Boss, B. Rappaz, C. Moratal, M. C. Hernandez, C. Depeursinge, P. J. Magistretti, and P. Marquet, "Simultaneous optical recording in multiple cells by digital holographic microscopy of chloride current associated to activation of the ligand-gated chloride channel GABA(A) receptor," Plos One 7, e51041 (2012).

8. R. A. Stepnoski, A. LaPorta, F. Raccuia-Behling, G. E. Blonder, R. E. Slusher, and D. Kleinfeld, "Noninvasive detection of changes in membrane potential in cultured neurons by light scattering," Proc Natl Acad Sci U S A 88, 9382-9386 (1991). 